\title{
Carbon Monoxide Vertical Column Density Retrieval from SCIAMACHY Infrared Nadir Observations
}

\author{
Franz Schreier, Sebastián Gimeno-García, M. Hess, A. Doicu, and G. Lichtenberg \\ DLR — Remote Sensing Technology Institute, Oberpfaffenhofen, 82234 Wessling, GERMANY
}

\begin{abstract}
Nadir observations in the shortwave infrared channels of SCIAMACHY onboard the ENVISAT satellite can be used to derive information on $\mathrm{CO}, \mathrm{CH}_{4}, \mathrm{~N}_{2} \mathrm{O}, \mathrm{CO}_{2}$, and $\mathrm{H}_{2} \mathrm{O}$. BIRRA (Beer InfraRed Retriaval Algorithm) is a nonlinear least squares fit of the measured radiance: Trace gas vertical profiles are scaled to fit the observed data, further auxiliary parameters are code dependent. Here we discuss some features of the code and present results of carbon monoxide vertical column densities retrieved from SCIAMACHY infrared nadir observations.
\end{abstract}

Keywords: Atmosphere, Remote Sensing, Radiative Transfer, ENVISAT

PACS: 92.60.H-, 93.85.Bc, 33.20.Ea, 02.30.Zz

\section{INTRODUCTION}

Nadir sounding of molecular column densities is well established in atmospheric remote sensing. For UV instruments such as SCIAMACHY [1] the analysis is traditionally based on a DOAS methodology. This approach has also been successfully applied to SCIAMACHY's near infrared channels [2,3]. To gain greater flexibility and an efficient and and to have a robust inversion for the operational level 2 data processing, "BIRRA" has been developed at DLR.

In the framework of the ongoing code verification, a careful intercomparison of BIRRA with independently developed codes, i.e., WFM-DOAS (developed by the University of Bremen [2]) and IMLM (SRON — Netherlands Institute for Space Research [4]) has been performed. Moreover, molecular column densities retrieved from infrared atmospheric soundings are compared. Results of carbon monoxide retrievals for 2004 are presented here.

\section{THEORY AND ALGORITHM}

The objective of SCIAMACHY nadir NIR measurements is to retrieve information on gases such as $\mathrm{N}_{2} \mathrm{O}, \mathrm{CH}_{4}$, or $\mathrm{CO}$, e.g., volume mixing ratio $q_{X}(z)$ or density $n_{X}(z)=q_{X}(z) \cdot n_{\text {air }}(z)$ of molecule $\mathrm{X}$. Unfortunately, the analysis of the NIR channels of SCIAMACHY is challenging because of

- Tiny signal on huge background

- Channel 8: ice layer on detector

- $\mathrm{CO}$ and $\mathrm{N}_{2} \mathrm{O}$ retrieval: very weak absorbers

Furthermore vertical sounding inversions are ill-posed, so it is customary to retrieve only column densities

$$
N_{X} \equiv \int_{z_{\text {ground }}}^{\infty} n_{x}(z) \mathrm{d} z
$$

\subsection{Near Infrared Radiative Transfer}

The intensity (radiance) $I$ at wavenumber $v$ received by an instrument at $s=0$ is described by the equation of radiative transfer [5]

$$
I(v)=I_{\mathrm{b}}(v) \mathscr{T}(v)-\int_{0}^{\infty} \mathrm{d} s^{\prime} J(v, s) \frac{\partial \mathscr{T}\left(v ; s^{\prime}\right)}{\partial s^{\prime}}
$$


where $I_{\mathrm{b}}$ is a background contribution and $J$ is the source function. In the near infrared, reflected sunlight becomes important, whereas thermal emission is neglegible. For clear sky observations scattering can be neglected, hence

$$
\begin{aligned}
I(v) & =r(v) I_{\text {sun }}(v) \mathscr{T}_{\uparrow}(v) \mathscr{T}_{\downarrow}(v) \\
& =r(v) I_{\text {sun }}(v) \exp \left[-\int_{z_{\mathrm{g}}}^{z_{\text {sat }}} \frac{\mathrm{d} z^{\prime}}{\mu} \sum_{m} \alpha_{m} \bar{n}_{m}\left(z^{\prime}\right) k_{m}\left(v, z^{\prime}\right)\right] \exp \left[-\int_{z_{\mathrm{g}}}^{\infty} \frac{\mathrm{d} z^{\prime \prime}}{\left|\mu_{\odot}\right|} \sum_{m} \alpha_{m} \bar{n}_{m}\left(z^{\prime \prime}\right) k_{m}\left(v, z^{\prime \prime}\right)\right]
\end{aligned}
$$

where $r$ is reflection (albedo) and $\mathscr{T}_{\uparrow}$ and $\mathscr{T}_{\downarrow}$ denote transmission between reflection point (e.g. Earth surface at altitude $\left.z_{\mathrm{b}}\right)$ and observer and between sun and reflection point, respectively. $k_{m}$ and $\bar{n}_{m}(z)$ are the (pressure and temperature dependent) absorption cross section and reference (e.g., climatological) density of molecule $m$, and $\alpha_{m}$ are the scale factors to be estimated. (Note that for simplicity we have used a plane-parallel approximation with $\mu \equiv \cos \theta$ for an observer zenith angle $\theta$ and $\mu_{\odot}$ for the solar zenith angle $\theta_{\odot}$; moreover continuum is neglected here.)

The instrument is taken into account by convolution of the monochromatic intensity spectrum (3) with an spectral response function $\mathscr{S}$ (e.g., Gaussian or hyperbolic with width $\gamma$ )

$$
\widetilde{I(v)} \equiv(I \otimes \mathscr{S})(v)=\int_{-\infty}^{\infty} I(v) \times \mathscr{S}\left(v-v^{\prime}\right) \mathrm{d} v^{\prime} .
$$

Introducing the total optical depth $\tau_{m}$ of molecule $m$, the forward model is written as (with an optional baseline $b$ )

$$
F(x) \equiv \widetilde{I(v)}=r I_{\text {sun }}(v) \exp \left[-\sum_{m} \alpha_{m} \tau_{m}(v)\right] \otimes \mathscr{S}(v, \gamma)+b
$$

The BIRRA forward model is based on MIRART (Modular IR $\underline{\text { Atmospheric }}$ Radiative Transfer) [6], a line-by-line code for arbitrary observation geometry (up, down, limb) and instrumental field-of-view and line shape that provides Jacobians by means of automatic differentation [7] and has been validated in extensive intercomparisons [e.g. 8, 9].

\subsection{The inverse problem - nonlinear least squares}

The standard approach to estimate the unknown $x$ from a measurement vector $y$ relies on (nonlinear) least squares

$$
\min _{x}\|y-F(x)\|^{2}
$$

Here $F$ denotes the forward model (5), and the unknown state vector $x$ is comprised of the geophysical and instrumental parameters $\alpha, \gamma, r$ and an optional additive baseline correction $b$ (more generally $r$ and $b$ are polynomials in wavenumber). For the nonlinear least squares problem (6) BIRRA uses solvers of the PORT Optimization Library [10] based on a scaled trust region strategy. Optionally a least squares with simple bounds (to prevent, e.g., negative values for physical parameters) or a separable nonlinear least squares solver can be used.

To compensate for variations of the meteorological conditions (e.g., pressure) and for instrumental artefacts (e.g., calibration, slit function), the vertical column density of $\mathrm{CO}$ is derived as

$$
\mathrm{xCO} \equiv N_{\mathrm{CO}} \times \frac{\alpha_{\mathrm{CO}}}{\alpha_{\mathrm{CH} 4}}
$$

Furthermore, column densities are averaged in 1dg latitude/longitude bins with doubtful values rejected:

- PORT least squares converged with residual norm decreased

- No "forbidden" $x$ values

- Scaling factors: $0 \leq \alpha_{\mathrm{CO}} \leq 1.2$ and $0.7 \leq \alpha_{\mathrm{CH} 4}^{\prime} \leq 1.3$ ( $\alpha_{\mathrm{CH} 4}^{\prime}$ throughput corrected)

- Latitudes in interval $[80 \mathrm{~S}, 80 \mathrm{~N}]$, only overland 


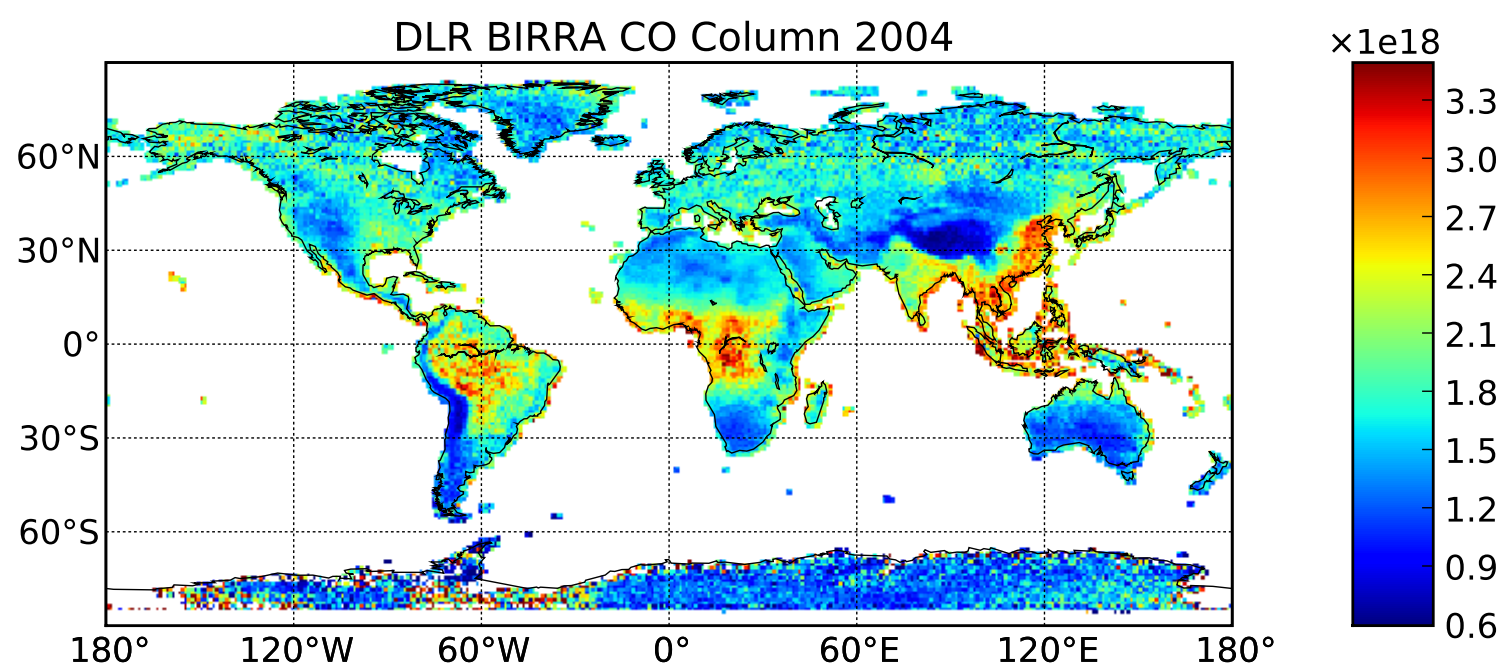

FIGURE 1. 2004 annual average of CO column densities retrieved with the BIRRA.

\section{RESULTS: CO VERTICAL COLUMN DENSITIES 2004}

Carbon monoxide is an important trace gas affecting air quality and climate and highly variable in space and time. About half of the $\mathrm{CO}$ comes from anthropogenic sources (e.g., fuel combustion), and further significant contributions are due to biomass burning. $\mathrm{CO}$ is a target species of several spaceborne instruments, nb. AIRS, MOPITT, and TES from NASA's EOS satellite series, and MIPAS and SCIAMACHY on ESA's Envisat.

For the retrieval of carbon monoxide vertical column densities with BIRRA, level 1 data of SCIAMACHY channel 8 applying the Bremen bad/dead pixel mask have been used; hence a single spectrum comprises 51 data points in the interval 4282.68615 to $4302.13102 \mathrm{~cm}^{-1}$. An US standard atmosphere was assumed. Surface reflectivity was modelled with a second order polynomial, baseline was ignored.

The annual average of $2004 \mathrm{CO}$ vertical columns is shown in Fig. 1. Intercomparisons of monthly means with other SCIAMACHY retrievals and with AIRS are shown in the subsequent figures. These months indicate local sources of $\mathrm{CO}$ associated with highly populated areas and biomass burning as well as seasonal variability.

\section{SUMMARY}

Carbon monoxide column densities retrieved with the "BIRRA" prototype of the operational SCIAMACHY near IR nadir level 2 processor have been compared with results obtained by the WFM-DOAS (University of Bremen) and IMLM (SRON) algorithms. Furthermore, intercomparisons with the CO product obtained with the Atmospheric Infrared Sounder (AIRS) have been shown. Results are highly sensitive to the bad/dead pixel mask and to the correct filtering of dubious retrieval results. Despite significant conceptual differences of the three SCIAMACHY codes w.r.t. forward modelling and inversion a good overall agreement has been found.

\section{ACKNOWLEDGMENTS}

We would like to thank Michael Buchwitz (IUP, University of Bremen) for many helpful discussions and for providing SCIAMACHY level 1 and level 2 (WFM-DOAS) data. Annemieke Gloudemans and Hans Schrijver (SRON) kindly delivered their SCIAMACHY carbon monoxide total column data. AIRS level 3 data (RETSDD029.v5) were retrieved from the NASA Langley Research Center Atmospheric Sciences Data Center. 

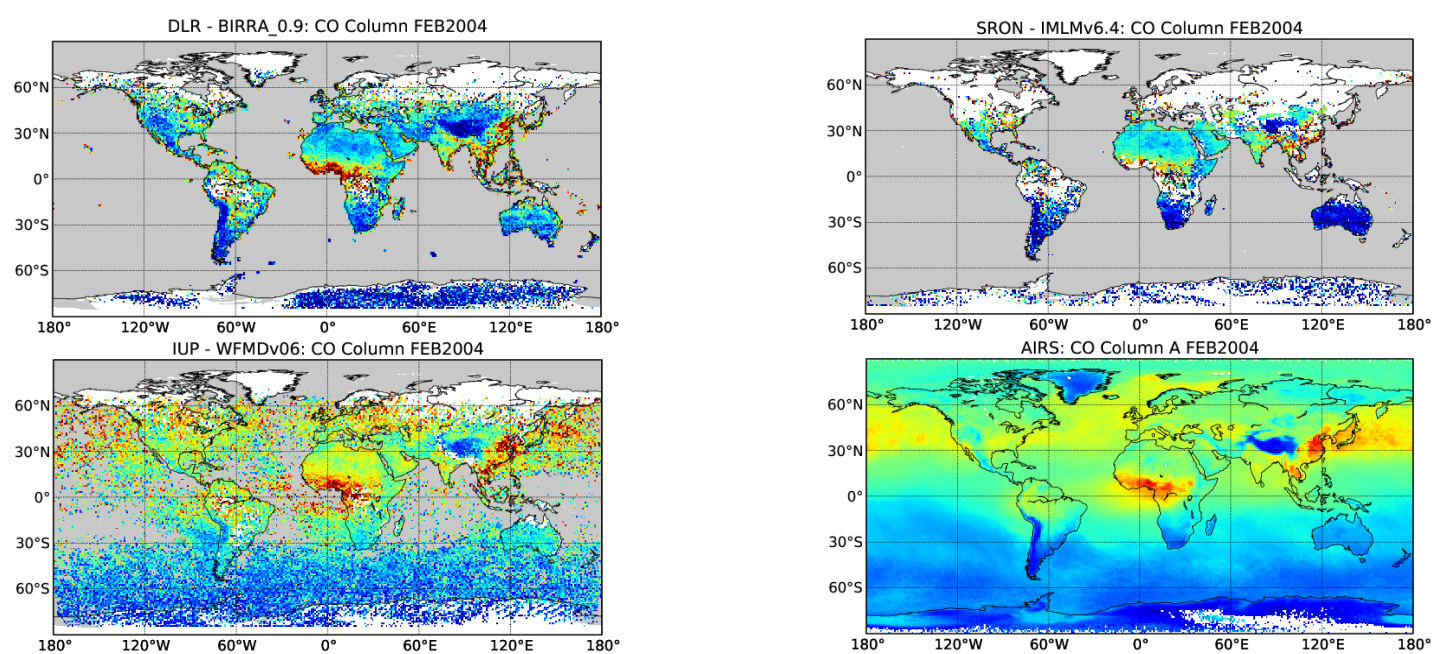

FIGURE 2. Comparison of February 2004 CO column densities. (Same color bar as in Fig. 1.)
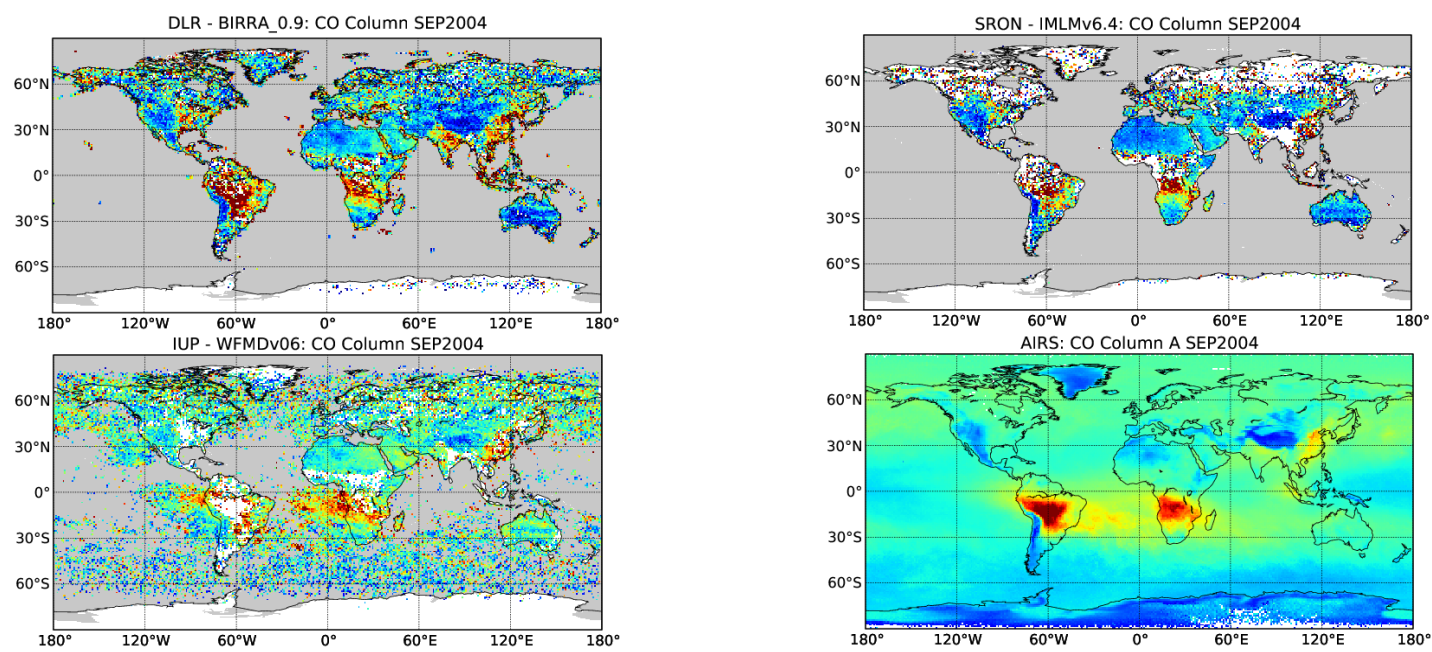

FIGURE 3. Comparison of September $2004 \mathrm{CO}$ column densities.

\section{REFERENCES}

1. H. Bovensmann, J. Burrows, M. Buchwitz, J. Frerick, S. Noël, V. Rozanov, K. Chance, and A. Goede, J. Atmos. Sci. 56, 127-150 (1999).

2. M. Buchwitz, I. Khlystova, H. Bovensmann, and J. P. Burrows, Atm. Chem. Phys. 7, 2399-2411 (2007).

3. C. Frankenberg, U. Platt, and T. Wagner, Atm. Chem. Phys. 5, 9-22 (2005).

4. A. Gloudemans, H. Schrijver, O. Hasekamp, and I. Aben, Atm. Chem. Phys. 8, 3999-4017 (2008).

5. K.-N. Liou, An Introduction to Atmospheric Radiation, Academic Press, Orlando, 1980.

6. F. Schreier, and B. Schimpf, "A New Efficient Line-By-Line Code for High Resolution Atmospheric Radiation Computations incl. Derivatives," in IRS 2000: Current Problems in Atmospheric Radiation, edited by W. Smith, and Y. Timofeyev, A. Deepak Publishing, 2001, pp. 381-384.

7. A. Griewank, Evaluating Derivatives: Principles and Techniques of Algorithmic Differentiation, SIAM, 2000.

8. T. von Clarmann, M. Höpfner, B. Funke, M. López-Puertas, A. Dudhia, V. Jay, F. Schreier, M. Ridolfi, S. Ceccherini, B. Kerridge, J. Reburn, and R. Siddans, J. Quant. Spectrosc. \& Radiat. Transfer 78, 381-407 (2002).

9. C. Melsheimer, C. Verdes, S. Bühler, C. Emde, P. Eriksson, D. Feist, S. Ichizawa, V. John, Y. Kasai, G. Kopp, N. Koulev, T. Kuhn, O. Lemke, S. Ochiai, F. Schreier, T. Sreerekha, M. Suzuki, C. Takahashi, S. Tsujimaru, and J. Urban, Radio Science 40, RS1007 (2005).

10. J. Dennis, Jr., D. Gay, and R. Welsch, ACM Trans. Math. Soft. 7, 348-368 (1981). 